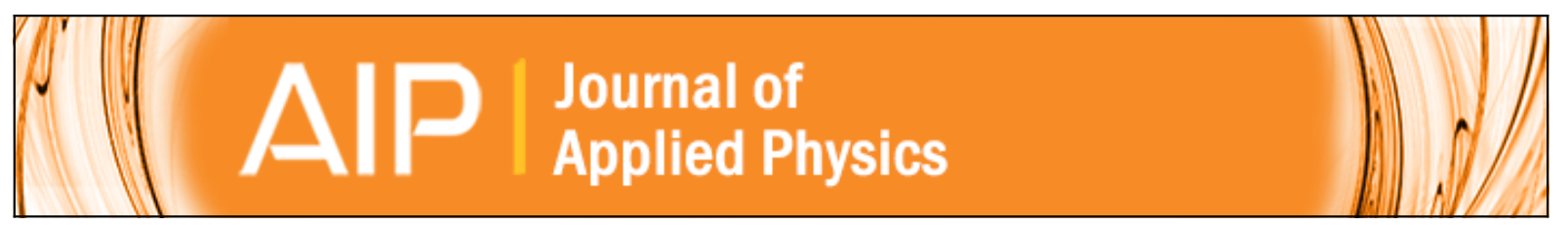

\title{
Surface effect on resonant properties of nanowires predicted by an elastic theory for
} nanomaterials

Yin Yao and Shaohua Chen

Citation: Journal of Applied Physics 118, 044303 (2015); doi: 10.1063/1.4927290

View online: http://dx.doi.org/10.1063/1.4927290

View Table of Contents: http://scitation.aip.org/content/aip/journal/jap/118/4?ver=pdfcov

Published by the AIP Publishing

\section{Articles you may be interested in}

Atomistic simulation for the size effect on the mechanical properties of $\mathrm{Ni} / \mathrm{Ni3Al}$ nanowire

J. Appl. Phys. 114, 094303 (2013); 10.1063/1.4820257

Effect of surface stress on the stiffness of micro/nanocantilevers: Nanowire elastic modulus measured by nanoscale tensile and vibrational techniques

J. Appl. Phys. 113, 013508 (2013); 10.1063/1.4772649

Probing the three-dimensional strain inhomogeneity and equilibrium elastic properties of single crystal $\mathrm{Ni}$ nanowires

Appl. Phys. Lett. 101, 033107 (2012); 10.1063/1.4737440

Characterization of surface and nonlinear elasticity in wurtzite $\mathrm{ZnO}$ nanowires

J. Appl. Phys. 111, 124305 (2012); 10.1063/1.4729545

Transverse resonant properties of strained gold nanowires

J. Appl. Phys. 108, 034318 (2010); 10.1063/1.3460127

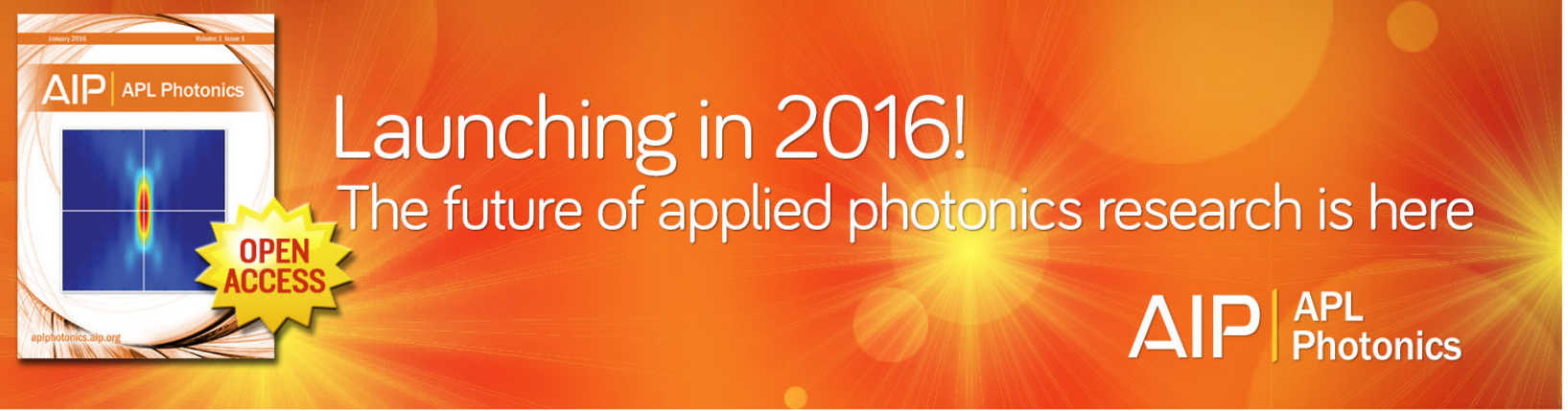




\title{
Surface effect on resonant properties of nanowires predicted by an elastic theory for nanomaterials
}

\author{
Yin Yao and Shaohua Chen ${ }^{\text {a) }}$ \\ LNM, Institute of Mechanics, Chinese Academy of Sciences, Beijing 100190, China
}

(Received 29 April 2015; accepted 12 July 2015; published online 22 July 2015)

\begin{abstract}
A recently developed continuum theory considering surface effect in nanomaterials is adopted to investigate the resonant properties of nanowires with different boundary conditions in the present paper. The main feature of the adopted theory is that the surface effect in nanomaterials is characterized by the surface energy density of the corresponding bulk materials and the surface relaxation parameter in nanoscale. Based on a fixed-fixed beam model and a cantilever one, the governing equation of resonant frequency for corresponding nanowires is obtained. Numerical calculation of the fundamental resonant frequency is carried out, the result of which is well consistent with the existing numerical ones. Comparing to the result predicted by the conventionally structural dynamics, the resonant frequency of a fixed-fixed nanowire is improved, while that of a cantilever nanowire is weakened due to the surface effect. Both a decreasing characteristic size (height or diameter) and an increasing aspect ratio could further enhance the varying trend of resonant properties for both kinds of nanowires. The present result should be helpful for the design of nano-devices and nanostructures related to nanowires. (C) 2015 AIP Publishing LLC.
\end{abstract}

[http://dx.doi.org/10.1063/1.4927290]

\section{INTRODUCTION}

Due to a large surface-to-volume ratio, the elastic property of nanowires exhibits a strong size-dependence, ${ }^{1-3}$ which is often measured and accessed by static bending experiments. It is found that the effective Young's modulus of a fixed-fixed nanowire shows an oppositely sizedependent behavior to that of a cantilever one..$^{3-8}$ It increases with a decreasing characteristic size for fixed-fixed nanowires, while decreases for cantilever ones. Such a behavior cannot be predicted by the classical beam theory, a proper one considering surface effect should be developed.

Surface elasticity theory ${ }^{9,10}$ has been widely adopted to characterize the elastic behavior of static-bending nanowires. ${ }^{111-15}$ Size-dependently effective Young's modulus of nanowires has been achieved, which depends on the surface elastic modulus. ${ }^{1,13}$ In addition, molecular dynamics (MD) simulation, as a major numerical approach, has also been adopted to study the size effect of nanowires' elastic properties. ${ }^{16-19}$

In nano-devices and nano-electro-mechanical systems (NEMs), nanowires are often used as resonant flexural structures, such as chemical and biological sensors and high frequency resonators. ${ }^{20-23}$ Size dependent elastic modulus of nanowires should induce a different resonant frequency from that of a corresponding bulk solid. In fact, resonance measurement is more convenient to obtain nanowire's elastic parameters than the static bending experiment due to a reduced amount of nanowire manipulation. ${ }^{24}$ The effective Young's moduli of silicon, gallium nitride $(\mathrm{GaN})$, silicon nitride $\left(\mathrm{SiN}_{\mathrm{x}}\right)$, and zinc oxide $(\mathrm{ZnO})$ cantilever nanowires

\footnotetext{
a) Author to whom correspondence should be addressed. Electronic addresses: chenshaohua72@hotmail.com and shchen@LNM.imech.ac.cn. Tel.: 86-10-82543960. Fax: 86-10-82543977.
}

were achieved experimentally by measuring the resonant frequency of vibrating nanowires. ${ }^{25-29}$ Numerically, Park and Klein $^{24}$ and Park ${ }^{30}$ calculated the resonant frequency of fixed-fixed nanowires and cantilevered ones with a surface Cauchy-Born (SCB) model, in which it was found that not only a decreasing height but also an increasing aspect ratio can enhance the surface effect on the resonant property. A finite element (FE) model was subsequently developed by Feng et al. ${ }^{31}$ in which it was found that the resonant frequency of a silicon nano-cantilever decreases with a decreasing height. MD simulation confirmed the enhancement of the resonant frequency of fixed-fixed nanowires in contrast to that of a bulk solid. ${ }^{32}$

With regard to the theoretical study on the resonant frequency of nanowires, Lu et al. ${ }^{33}$ pointed out that the straindependent surface stress has a considerable influence on the resonant frequency of nano-cantilevers. Wang and Feng ${ }^{34}$ proposed first a generalized Young-Laplace (Y-L) model to study effects of surface elasticity and residual surface tension on the natural frequency of nanowires. Subsequently, He and Lilley ${ }^{35}$ adopted the Y-L model to analyze the resonant bending behavior of nanowires with different boundary conditions. The fundamental resonant frequency of both fixed-fixed nanowires and cantilevered ones exhibits the same varying trend as that of the effective elastic moduli. ${ }^{12}$ Later, the Y-L model was extended to the vibration problem of Timoshenko nanowires. ${ }^{36,37}$ Chiu and $\mathrm{Chen}^{38}$ further discussed the effect of the surface bending modulus on the resonant frequency of nanowires. Recently, Zhang et al. ${ }^{39}$ analyzed the motion equation of vibrating nanowires, based on which contributions of surface elasticity and surface stress to the resonant frequency can be abstracted, respectively.

Surface elasticity theory, as the most popular theoretical approach, is always adopted to investigate surface effect of 
nanowires, ${ }^{40}$ in which surface elastic constants, including the surface elastic modulus and surface bending modulus, serve as critical parameters influencing the size-dependent behavior of nanowires. ${ }^{12,13,31,35,38}$ However, surface elastic constants needed by the surface elasticity theory can only be provided by MD simulation and hardly be measured by proper experiment techniques. ${ }^{1,13,41,42}$ How to define the atomic layer as a surface layer, how to choose a proper atomic interaction potential and the size of a numerical model are still open questions in MD simulation.

Recently, an elastic theory for nanomaterials was developed, ${ }^{43}$ in which only the surface energy density of a bulk solid and the surface relaxation parameter in nanoscale are needed to characterize the surface effect in nanomaterials without introducing surface elastic constants. Such a new theory has been adopted to predict successfully the mechanical behavior of nanofilms subjected to a bi-axial tension, size effect of the surface energy density of nanoparticles and the static bending behavior of nanowires. ${ }^{43-45}$ All the theoretical predictions agree well with the experimental and numerical results. ${ }^{3-5,7,46-49}$

In the present paper, the resonant vibration of both fixed-fixed nanowires and cantilevered ones is investigated with the new developed elastic theory for nanomaterials and theoretical predictions are further compared with the existing numerical results. Geometrical factors governing the size dependent characteristics of resonant frequency are also discussed, which could provide a theoretical guidance for the design of nanowire resonators and sensors in NEMs.

\section{BRIEF INTRODUCTION OF THE NEW DEVELOPED THEORY FOR NANOMATERIALS}

An elastic theory based on the surface energy density to characterize the size effect in nanomaterials has been proposed by Chen and Yao. ${ }^{43}$ Consider a nano-solid with an idealized crystal structure in an initial (or reference) configuration. A Lagrangian coordinate system is imbedded in the crystal surface and attached to surface atoms, ${ }^{50}$ with principal axes 1 and 2 parallel to the two basic vectors of the surface unit cell as shown in Figure 1. $a_{01}, a_{02}$ represent the lattice lengths in the two principal directions, respectively. Due to a surface relaxation, the lattice lengths become $a_{r 1}$ and $a_{r 2}$, and further become $a_{1}$ and $a_{2}$ in the current configuration subjected to an external loading. $\beta$ denotes an angle between the two basic vectors.

The potential energy function $\Pi$ of a nano-solid in the current configuration can be written as

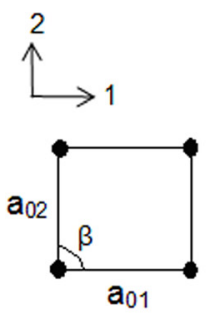

initial configuration
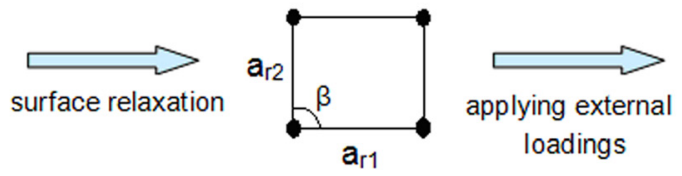

relaxed configuration

$$
\Pi(\boldsymbol{u})=\int_{V-S} \psi(\boldsymbol{\varepsilon}) d V+\int_{S} \phi d S-\int_{V-S} \boldsymbol{f} \cdot \boldsymbol{u} d V-\int_{S_{p}} \boldsymbol{p} \cdot \boldsymbol{u} d S
$$

where $\psi$ is the elastic strain energy density, $\boldsymbol{f}$ and $\boldsymbol{p}$ are the body force and external surface traction, respectively. $\boldsymbol{u}$ and $\boldsymbol{\varepsilon}$ are the displacement and strain induced by $\boldsymbol{f}$ and $\boldsymbol{p}$. Variation analysis of Eq. (1) yields the following equilibrium equations and stress boundary conditions:

$$
\left\{\begin{array}{l}
\boldsymbol{\sigma} \cdot \nabla+\boldsymbol{f}=0 \quad(\text { in } V-S) \\
\boldsymbol{n} \cdot \boldsymbol{\sigma} \cdot \boldsymbol{n}=\boldsymbol{p} \cdot \boldsymbol{n}-\gamma_{n} \boldsymbol{n} \quad(\text { on } S) \\
(\boldsymbol{I}-\boldsymbol{n} \otimes \boldsymbol{n}) \cdot \boldsymbol{\sigma} \cdot \boldsymbol{n}=(\boldsymbol{I}-\boldsymbol{n} \otimes \boldsymbol{n}) \cdot \boldsymbol{p}-\gamma_{t} \quad(\text { on } S),
\end{array}\right.
$$

where $\boldsymbol{\sigma}$ is the bulk Cauchy stress tensor, $\boldsymbol{n}$ is the unit normal vector perpendicular to the boundary surface $S$ of the nanosolid, $\boldsymbol{I}$ is a unit tensor; $\gamma_{n}$ and $\gamma_{t}$ are the normal and tangential components of an additionally surface-induced traction vector, respectively, which characterizes force disturbances at boundaries due to the surface effect. Based on an infinitesimal element, the virtual work method yields the surface-induced traction, which is related to the surface energy density, ${ }^{43}$

$$
\gamma_{t}=\nabla_{s} \phi, \gamma_{n} \boldsymbol{n}=\phi\left(\frac{1}{R_{1}}+\frac{1}{R_{2}}\right) \boldsymbol{n}=\phi\left(\boldsymbol{n} \cdot \nabla_{s}\right) \boldsymbol{n},
$$

where $\nabla_{s}$ is a surface gradient operator, $\phi$ is the Eulerian surface-energy density in the current configuration, $R_{1}$ and $R_{2}$ are two principal radii of curvature of a curved surface.

Relation between the Eulerian surface-energy density $\phi$ in the current configuration and the Lagrangian surface energy density $\phi_{0}$ in the reference configuration satisfies

$$
\phi=\frac{\phi_{0}}{J_{s}},
$$

where $J_{s}$ is a Jacobean determinant characterizing the surface deformation from the reference configuration to the current one. Equation (4) can also be found in Nix and $\mathrm{GaO}^{50}$ and Huang and Wang. ${ }^{51}$ Combining Eqs. (2)-(4), the equilibrium equation can be rewritten as, ${ }^{43}$

$$
\left\{\begin{array}{l}
\boldsymbol{\sigma} \cdot \nabla+\boldsymbol{f}=0(\text { in } V-S) \\
\boldsymbol{n} \cdot \boldsymbol{\sigma} \cdot \boldsymbol{n}=\boldsymbol{p} \cdot \boldsymbol{n}-\frac{\phi_{0}\left(\boldsymbol{n} \cdot \nabla_{s}\right)}{J_{s}}(\text { on } S) \\
(\boldsymbol{I}-\boldsymbol{n} \otimes \boldsymbol{n}) \cdot \boldsymbol{\sigma} \cdot \boldsymbol{n}=(\boldsymbol{I}-\boldsymbol{n} \otimes \boldsymbol{n}) \cdot \boldsymbol{p}+\frac{\phi_{0}\left(\nabla_{s} J_{s}\right)}{J_{s}^{2}}-\frac{\nabla_{s} \phi_{0}}{J_{s}}(\text { on } S) .
\end{array}\right.
$$

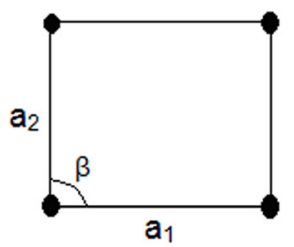

current configuration
FIG. 1. Schematic of a surface unit cell in the initial (reference), relaxed, and current configurations. 
The Lagrangian surface energy density $\phi_{0}$ in the reference configuration can be divided into a structural part $\phi_{0}^{\text {stru }}$ related to the surface strain energy and a chemical part $\phi_{0}^{\text {chem }}$ originating from the surface dangling-bond energy, ${ }_{43,52,53}$

$$
\begin{aligned}
\phi_{0}= & \phi_{0}^{\text {stru }}+\phi_{0}^{\text {chem }}, \\
\phi_{0}^{\text {stru }}= & \frac{E_{b}}{2 \sin \beta} \sum_{i=1}^{2} a_{0 i} \eta_{i}\left\{\left[3+\left(\lambda_{i}+\lambda_{i} \varepsilon_{s i}\right)^{-m}-3\left(\lambda_{i}+\lambda_{i} \varepsilon_{s i}\right)\right]\right. \\
& \left.\times\left[\lambda_{i}^{2} \varepsilon_{s i}^{2}+\left(\lambda_{i}-1\right)^{2}+2 \lambda_{i}\left(\lambda_{i}-1\right) \varepsilon_{s i}\right]\right\} \\
\phi_{0}^{\text {chem }}= & \phi_{0 b}\left(1-\frac{D_{0}}{w_{1} D}\right), \quad \eta_{1}=a_{01} / a_{02}, \eta_{2}=a_{02} / a_{01},
\end{aligned}
$$

where $\phi_{0 b}$ is the constant surface energy density of bulk solids, $D_{0}$ is a critical size $\left(D_{0}=3 d_{a}\right.$ for nanoparticles, nanowires, and $2 d_{a}$ for nano-thin films, where $d_{a}$ is the atomic diameter). $D$ is a characteristic scale of nanomaterials (e.g., thickness, diameter, etc.). $w_{1}$ is a parameter governing the size-dependent behavior of $\phi_{0}^{\text {chem }} . E_{b}$ is the constant Young's modulus of bulk solids, $\lambda_{i}=a_{r i} / a_{0 i}$ denotes the surface relaxation parameter, $\varepsilon_{s i}=\left(a_{i}-a_{r i}\right) / a_{r i}$ is the surface strain induced only by the external loading; $m$ is a parameter describing the dependence of bond lengths on the binding energy ( $m=4$ for alloys or compounds and $m=1$ for pure metals). ${ }^{52}$ Detailed derivations of Eq. (6) can be found in Ref. 43.

In contrast to the surface elasticity theory, ${ }^{9}$ the new developed theory no longer requires the surface elastic constants. The Lagrangian surface-energy density $\phi_{0}$ in the reference configuration serves as a unique quantity characterizing the surface effect of nanomaterials, which depends on the bulk surface-energy density and the relaxation parameter. Both parameters have clearly physical meanings and are very easy to be determined through experiment and simple MD simulation, which show the main advantage of our model as compared with the surface elasticity one. The bulk surface energy density is a constant for a given material. The surface relaxation parameter depending on the characteristic size of nanomaterials directly affects the Lagrangian surface energy density.

\section{SURFACE EFFECTS IN VIBRATING NANOWIRES}

The new theory for nanomaterials is used to analyze the resonant bending of nanowires as shown in Fig. 2, where the length of the nanowire in the $x$ direction is $L$ and the vertical deflection in $z$ direction is $w$. The cross-section of the nanowire is rectangular with a height $h$ and a width $b(b \geq h)$, or circular with a diameter $d$. Two kinds of boundary value problems, i.e., a cantilever beam and a fixed-fixed one, will be investigated, respectively. In resonant bending tests, the vertical deflection of a nanowire is induced by a thermal noise spectra $^{27}$ or an electric field, ${ }^{28}$ which depends not only on the coordinate $x$ but also on the time variable $t$, i.e., $w=w(x, t)$.

\section{A. Variational analysis}

According to the assumption of Euler-Bernoulli beam, the axial and vertical displacements $u_{x}, u_{z}$ and the axial strain $\varepsilon_{x}$ of the nanowire can be expressed as, ${ }^{54}$

$$
\begin{aligned}
u_{x} & =-z \frac{\partial w}{\partial x}, \quad u_{z}=w(x, t), \quad \varepsilon_{x}=-z \frac{\partial^{2} w}{\partial x^{2}}, \\
0 & \leq x \leq L, 0 \leq t \leq T .
\end{aligned}
$$

It is well known that the surface property of a nanoscale system is highly facet-dependent, ${ }^{55}$ leading to different Lagrangian surface energy densities on different crystal faces. For simplicity, we assume a [100] axially oriented nanowire with a symmetric lateral surface in the present model, which has an equal atom spacing in both bond directions, e.g., the $(001)$ or $(010)$ surface. ${ }^{13,34,35}$ In such a case, the Lagrangian surface energy density can be written as ${ }^{45}$

$$
\begin{aligned}
\phi_{0}= & \phi_{0 b}\left(1-\frac{3 d_{a}}{4 D}\right)+\frac{\sqrt{2} E_{b} a_{0}}{2}\left[3+\frac{1}{\lambda\left(1+\varepsilon_{x} / 2\right)}\right. \\
& \left.-3\left(\lambda+\frac{\lambda \varepsilon_{x}}{2}\right)\right]\left[\frac{\lambda^{2} \varepsilon_{x}^{2}}{4}+2 \lambda(\lambda-1) \frac{\varepsilon_{x}}{2}+(\lambda-1)^{2}\right],
\end{aligned}
$$

where $\lambda$ and $a_{0}$ represent the surface relaxation parameter in both bond directions of (001) surface and the bulk lattice constant, respectively; ${ }^{53} \mathrm{D}$ can be taken as the diameter or height of nanowires.

The variation of bulk strain energy is written as

$$
\begin{aligned}
\delta U & =\delta \int_{0}^{T} d t \int_{V} \frac{1}{2} \sigma_{x} \varepsilon_{x} d V=\delta \int_{0}^{T} d t \int_{0}^{L} \frac{1}{2} E_{b} I\left(\frac{\partial^{2} w}{\partial x^{2}}\right)^{2} d x \\
& =\int_{0}^{T} d t \int_{0}^{L} E_{b} I \frac{\partial^{2} w}{\partial x^{2}} \frac{\partial^{2}(\delta w)}{\partial x^{2}} d x
\end{aligned}
$$

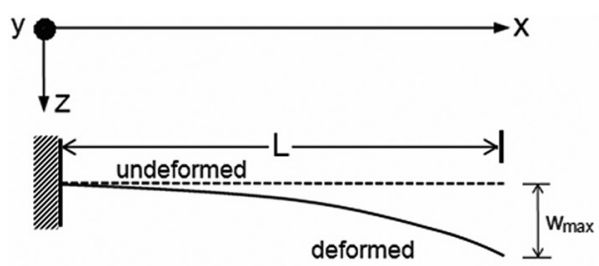

(a)
Cantilever

(c)

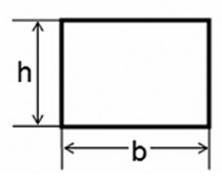

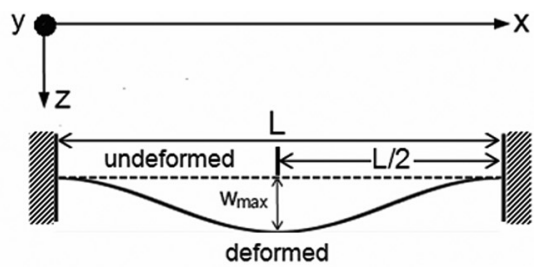

(b)
FIG. 2. Theoretical model of two kinds of nanowires. (a) A cantilever nanowire; (b) a fixed-fixed nanowire; (c) the cross-section of the nanowire. 
where $I=\int_{A_{n w}} z^{2} d A$ is the inertia moment, $A_{n w}$ denotes the nanowire's cross-section, and $E_{b}$ is the bulk Young's modulus.

The variation of surface energy can be written as ${ }^{56}$

$\delta \Phi=\int_{0}^{T} d t \int_{S_{n w}} \boldsymbol{\gamma} \cdot \delta \boldsymbol{u} d S=\int_{0}^{T} \int_{0}^{L} d x d t \int_{C_{n w}}\left(\gamma_{x} \delta u_{x}+\gamma_{n} \delta u_{n}\right) d C$,

where $S_{n w}$ represents the surface of nanowires, $C_{n w}$ is the perimeter of a rectangular or a circular cross-section. $\gamma_{x}$ and $\gamma_{n}$ represent the axial and normal components of the surfaceinduced traction, respectively. $\delta u_{x}$ and $\delta u_{n}$ are the corresponding displacement components of $\delta \boldsymbol{u}$. Combining Eqs. (3), (4), (7), and (8) and noting that $J_{s}=\lambda^{2}\left(1+\varepsilon_{x} / 2\right)^{2}$ yield the axially surface-induced traction $\gamma_{x},{ }^{45}$

$$
\begin{aligned}
\gamma_{x} & =\left[C_{0} z+C_{1} z^{2} \frac{\partial^{2} w}{\partial x^{2}}+C_{2} z^{3}\left(\frac{\partial^{2} w}{\partial x^{2}}\right)^{2}\right] \frac{\partial^{3} w}{\partial x^{3}}, \\
C_{0} & =\phi_{0}^{*}(5-4 \lambda)-\frac{\sqrt{2} E_{b} a_{0} A_{2}(3-2 \lambda)}{2}, \\
C_{1} & =2 \phi_{0}^{*}+\sqrt{2} E_{b} a_{0} A_{1}(3-2 \lambda)-\frac{\sqrt{2} E_{b} a_{0} A_{2}(5-4 \lambda)}{2}, \\
C_{2} & =\frac{\sqrt{2} E_{b} a_{0} A_{1}(7-4 \lambda)}{2}-\sqrt{2} E_{b} a_{0} A_{2}, \\
\phi_{0}^{*} & =\phi_{0 b}\left(1-\frac{3 d_{a}}{4 D}\right)+\frac{\sqrt{2} E_{b} a_{0}}{2}(\lambda-1)^{2}, \\
A_{1} & =\frac{1-10(\lambda-1)-17(\lambda-1)^{2}}{4}, \\
A_{2} & =(\lambda-1)-5(\lambda-1)^{2} .
\end{aligned}
$$

Noting the curvature $\kappa=-\left(\boldsymbol{n} \cdot \nabla_{s}\right)=d^{2} w / d x^{2}$ and $\delta u_{n} \approx \delta w,{ }^{57,58}$ the energy variation induced by the normal surface-induced traction $\gamma_{n}$ can be expressed as

$$
\begin{aligned}
\gamma_{n} \delta u_{n} \approx & -\phi \kappa \delta w \\
= & -\left[D_{0} z+D_{1} z \frac{\partial^{2} w}{\partial x^{2}}+D_{2} z^{2}\left(\frac{\partial^{2} w}{\partial x^{2}}\right)^{2}\right. \\
& \left.+D_{3} z^{3}\left(\frac{\partial^{2} w}{\partial x^{2}}\right)^{3}\right] \frac{\partial^{2} w}{\partial x^{2}} \delta w \\
D_{0}= & \phi_{0}^{*}(3-2 \lambda), \quad D_{1}=\phi_{0}^{*}-\frac{\sqrt{2} E_{b} a_{0} A_{2}(3-2 \lambda)}{2}, \\
D_{2}= & \frac{\sqrt{2} E_{b} a_{0} A_{1}(3-2 \lambda)}{2}-\sqrt{2} E_{b} a_{0} A_{2}, \\
D_{3}= & \frac{\sqrt{2} E_{b} a_{0} A_{1}}{2} .
\end{aligned}
$$

The variation of kinetic energy is

$$
\begin{aligned}
\delta K & =\delta \int_{0}^{T} d t \int_{V} \frac{1}{2} \rho\left(\frac{\partial w}{\partial t}\right)^{2} d V=\delta \int_{0}^{T} d t \int_{0}^{L} \frac{1}{2} \rho A_{n w}\left(\frac{\partial w}{\partial t}\right)^{2} d x \\
& =\int_{0}^{T} \int_{0}^{L} \rho A_{n w} \frac{\partial^{2} w}{\partial t^{2}} \delta w d x d t
\end{aligned}
$$

where $\rho$ is the mass density of bulk materials; the crosssection $A_{n w}=b h$ is for a rectangular nanowire and
$A_{n w}=\pi d^{2} / 4$ for a circular one. Combining Eqs. (9)-(13) leads to the variation of potential energy function,

$$
\begin{aligned}
\delta \Pi= & \delta U+\delta \Phi+\delta K \\
= & \int_{0}^{T} \int_{0}^{L} E_{b} I\left(\frac{\partial^{2} w}{\partial x^{2}}\right) \frac{\partial^{2}(\delta w)}{\partial x^{2}} d x d t \\
& -\int_{0}^{T} \int_{0}^{L}\left[C_{0} I_{s 1}+C_{2} I_{s 2}\left(\frac{\partial^{2} w}{\partial x^{2}}\right)^{2}\right] \frac{\partial^{3} w}{\partial x^{3}} \frac{\partial(\delta w)}{\partial x} d x d t \\
& -\int_{0}^{T} \int_{0}^{L}\left[D_{0} I_{c}+D_{2} I_{s 1}\left(\frac{\partial^{2} w}{\partial x^{2}}\right)^{2}\right] \frac{\partial^{2} w}{\partial x^{2}} \delta w d x d t \\
& +\int_{0}^{T} \int_{0}^{L} \rho A_{n w} \frac{\partial^{2} w}{\partial t^{2}} \delta w d x d t
\end{aligned}
$$

in which $I_{s 1}=\int_{C_{N W}} z^{2} d C, I_{s 2}=\int_{C_{N W}} z^{4} d C$, and $I_{c}=\int_{C_{n w}} n_{w}^{2} d C$. Here, $n_{w}$ represents the vertical component of the unit normal vector $\boldsymbol{n}$, which is parallel to $w(x, t)$.

For the case with different cross-section shapes, we have

Rectangular: $I=\frac{b h^{3}}{12}, \quad I_{s 1}=\frac{b h^{2}}{2}+\frac{h^{3}}{6}$,

$$
I_{s 2}=\frac{b h^{4}}{8}+\frac{h^{5}}{80}, \quad I_{c}=2 b,
$$

Circular : $I=\frac{\pi d^{4}}{64}, \quad I_{s 1}=\frac{\pi d^{3}}{8}, \quad I_{s 1}=\frac{3 \pi d^{5}}{128}, \quad I_{c}=\frac{\pi d}{2}$,

where the height $h$ of a rectangular cross-section and the diameter $d$ of a circular one are always considered to possess a nanometer scale. Variational analysis of Eq. (14) leads to the equilibrium equation of a vibrating nanowire (the highorder partial derivative of $w$ is ignored),

$$
\left(E_{b} I+C_{0} I_{s 1}\right) \frac{\partial^{4} w}{\partial x^{4}}-D_{0} I_{c} \frac{\partial^{2} w}{\partial x^{2}}=-\rho A_{n w} \frac{\partial^{2} w}{\partial t^{2}} .
$$

Let $w(x, t)=W(x) T(t)$ and $T(t)=T_{1} \cos (\omega t)+T_{2} \sin (\omega t),{ }^{59}$ where $\omega$ is the angular resonant frequency of nanowires and the resonant frequency $f=\omega / 2 \pi$. Then, Eq. (16) can be further reduced to an ordinary differential equation with respect to the vertical displacement function $W(x)$,

$$
\left(E_{b} I+C_{0} I_{s 1}\right) \frac{d^{4} W}{d x^{4}}-D_{0} I_{c} \frac{d^{2} W}{d x^{2}}=\rho A_{n w} \omega^{2} W(x) .
$$

\section{B. Solution of a fixed-fixed nanowire}

Solving Eq. (17) yields the general solution of $W(x)$,

$$
\begin{aligned}
W(x)= & W_{1} \cos \left(k_{1} x\right)+W_{2} \sin \left(k_{1} x\right)+W_{3} \cosh \left(k_{2} x\right) \\
& +W_{4} \sinh \left(k_{2} x\right)
\end{aligned}
$$

where $W_{1} \sim W_{4}$ are undetermined coefficients and the parameters $k_{1}, k_{2}$ satisfy 


$$
\begin{aligned}
& k_{1}=\sqrt{\sqrt{\left(\frac{c_{1}}{2 L^{2}}\right)^{2}+\left(\frac{\omega}{c_{2}}\right)^{2}}-\frac{c_{1}}{2 L^{2}}}, \\
& k_{2}=\sqrt{\sqrt{\left(\frac{c_{1}}{2 L^{2}}\right)^{2}+\left(\frac{\omega}{c_{2}}\right)^{2}}+\frac{c_{1}}{2 L^{2}}}, \\
& c_{1}=\frac{D_{0} I_{c} L^{2}}{E_{b} I+C_{0} I_{s 1}}, \quad c_{2}=\sqrt{\frac{E_{b} I+C_{0} I_{s 1}}{\rho A_{n w}}},
\end{aligned}
$$

with the following boundary conditions:

$$
W(0)=0, \quad W^{\prime}(0)=0, \quad W(L)=0, \quad W^{\prime}(L)=0 .
$$

Combining Eqs. (18)-(20), the governing equation of the angular resonant frequency $\omega$ can be derived,

$$
\begin{aligned}
& k_{1} k_{2}\left[\cos \left(k_{1} L\right)-\cosh \left(k_{2} L\right)\right]^{2}+\left[k_{1} \sin \left(k_{1} L\right)+k_{2} \sinh \left(k_{2} L\right)\right] \\
& \quad \times\left[k_{2} \sin \left(k_{1} L\right)-k_{1} \sinh \left(k_{2} L\right)\right]=0 .
\end{aligned}
$$

\section{Solution of a cantilever nanowire}

For a cantilever nanowire, the general solution of $W(x)$ is the same as Eq. (18) but with different boundary conditions,

$$
W(0)=0, \quad W^{\prime}(0)=0, \quad W^{\prime \prime}(L)=0, \quad W^{\prime \prime \prime}(L)=0 .
$$

Combining Eqs. (18) and (22) leads to the governing equation of the angular resonant frequency $\omega$ for a cantilever nanowire,

$$
\begin{aligned}
& {\left[k_{1}^{2} \cos \left(k_{1} L\right)+k_{2}^{2} \cosh \left(k_{2} L\right)\right]^{2}+\left[k_{1} \sin \left(k_{1} L\right)+k_{2} \sinh \left(k_{2} L\right)\right]} \\
& \quad \times\left[k_{1}^{3} \sin \left(k_{1} L\right)-k_{2}^{3} \sinh \left(k_{2} L\right)\right]=0 .
\end{aligned}
$$

We note that both Eqs. (21) and (23) are transcendental equations having infinite number of roots. In the following research, only the smallest positive solution of the two equations is numerically solved, which is referred as the angular resonant frequency for the first vibration modes (fundamental resonant frequency). ${ }^{35,58}$ Meanwhile, according to the conventional structural dynamics, ${ }^{59}$ the fundamental frequency for a bulk solid is,

$$
f_{b}=\frac{\omega_{b}}{2 \pi}=\frac{\xi^{2}}{2 \pi L^{2}} \sqrt{\frac{E_{b} I}{\rho A_{n w}}},
$$

where $\xi=1.875$ for a cantilever beam and $\xi=4.73$ for a fixed-fixed beam.

\section{RESULT AND DISCUSSION}

The fundamental resonant frequency of a fixed-fixed gold nanowire and a cantilever one with (001) surface was calculated numerically by Park and Klein ${ }^{24}$ based on a SCB model, which is compared to the theoretical prediction by the present model and the Young-Laplace (Y-L) one ${ }^{35}$ as shown in Fig. 3. The axial length of the nanowire is $L=232 \mathrm{~nm}$ and the square cross-section has a side length $h$ ranging from 7 to
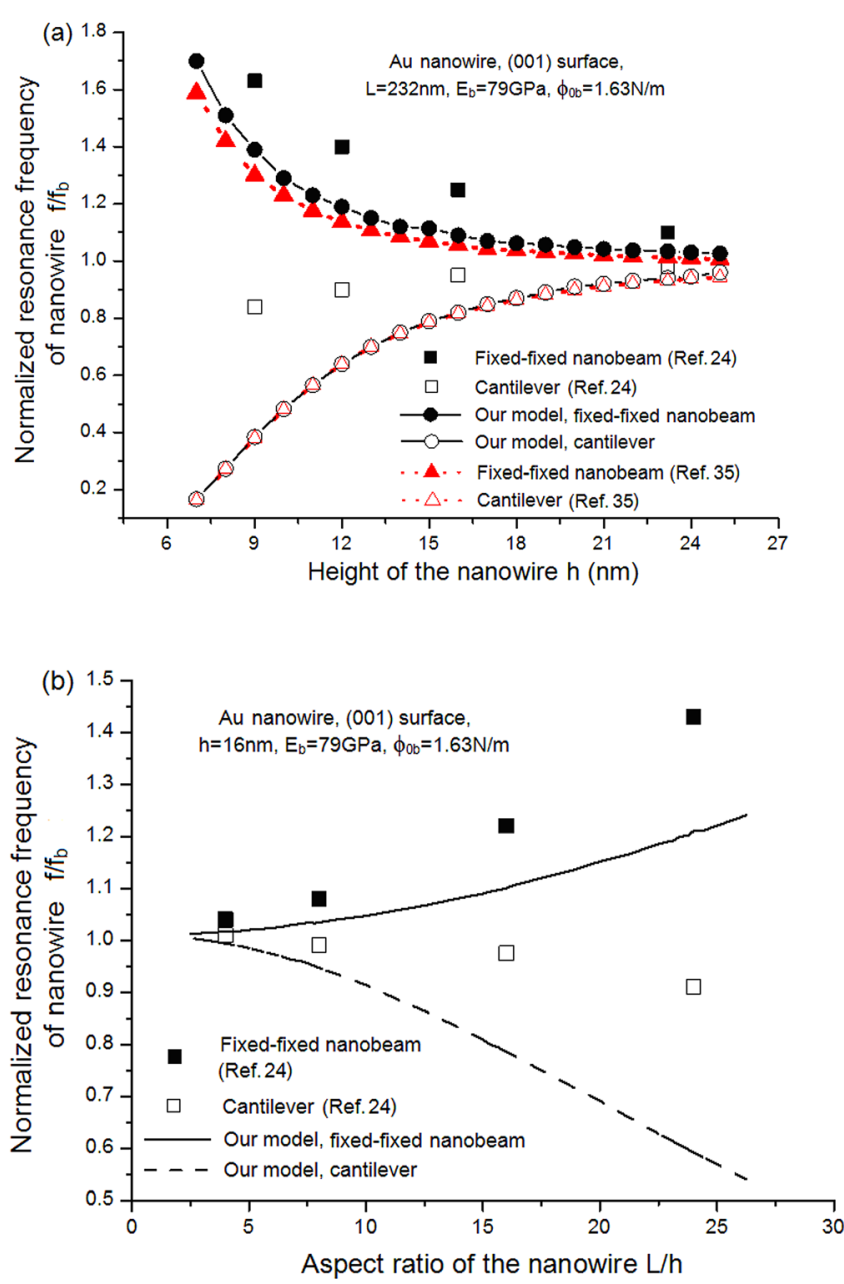

FIG. 3. Comparison between the theoretical prediction and the existing numerical result ${ }^{24}$ for a cantilever gold nanowire and a fixed-fixed one. (a) The resonant frequency is a function of the height of nanowires; (b) the resonant frequency is a function of the aspect ratio of nanowires.

$25 \mathrm{~nm}$. The mass density is $\rho=19.3 \times 10^{3} \mathrm{~kg} / \mathrm{m}^{3}$ and the surface elastic modulus $E_{S}$ in the $\mathrm{Y}-\mathrm{L}$ model is taken as $-3.6 \mathrm{~N} / \mathrm{m}^{41}$ The other material parameters involved in the model are $d_{a}=0.2884 \mathrm{~nm}, a_{0}=0.42 \mathrm{~nm}, E_{b}=79 \mathrm{GPa},{ }^{60}$ and $\phi_{0 b(001)}=1.63 \mathrm{~J} / \mathrm{m}^{2}$. ${ }^{61}$ The isotropic surface relaxation parameter $\lambda$ is proportional to the nano-scale height or diameter of nanowires, which can be expressed as $\lambda=1-c_{r} / D$ $\left(c_{r}>0, D=h\right.$ or $\left.d\right){ }^{32,62} c_{r} \approx 0.025 \mathrm{~nm}$ is determined for (001) gold surface. ${ }^{43}$ When the characteristic size is relatively large (for example, $D \geq 5 \mathrm{~nm}$ ), the surface relaxation parameter $\lambda$ tends to be unity.

Figure 3 shows that the theoretical prediction is qualitatively consistent with the numerical data given by Park and Klein. ${ }^{24}$ The normalized resonant frequency $f / f_{b}$ of a fixedfixed nanowire increases, while that of a nano-cantilever decreases with a decreasing height $h$ or an increasing aspect ratio $L / h$, which exhibits the same size-dependent behavior as the effective Young's modulus of nanowires since $\left(f / f_{b}\right)^{2}=E_{e f f} / E_{b} \cdot{ }^{12,24,35}$ Such a size effect of elastic modulus is basically induced by different deflection curvatures under different boundary conditions. ${ }^{45}$ As the height of nanowires increases or the aspect ratio decreases, the surface effect is weakened and both the theoretical result and the 
numerical one approach the bulk value. The classical elasticity theory cannot predict the size-dependent phenomena, while the theoretical model considering the size effect works, indicating the surface effect in nanomaterials arising from the sample geometry. Therefore, the vibrating frequency of resonators and sensors in NEMs can be effectively controlled by tailoring the geometrical size and boundary of nanowires. Comparing the result predicted by the Y-L model $^{35}$ and that given by the present model, good agreement can be found. However, determination of the surface elastic modulus is avoided in the present model. In addition, the bulk surface energy density and the surface relaxation parameter in the present continuum model can be easily achieved.

A quantitative difference can be found between our result and the one obtained by Park and Klein, ${ }^{24}$ especially for the case of cantilever nanowires with a small height or a large aspect ratio $(h \leq 12 \mathrm{~nm}$ or $L / h \geq 15)$. He and Lilley ${ }^{35}$ argued that such a difference is due to the axial relaxation of nanowires, which can be captured in the numerical model but not considered in the Y-L model. However, the surface relaxation of a gold nanowire was proved to almost vanish when the height is larger than $5 \mathrm{~nm},{ }^{32,62}$ which should have an insignificant effect on the mechanical performance of nanowires considered here $(h \geq 7 \mathrm{~nm})$. As shown in Fig. 4, the SCB model actually takes into account a surface region consisting of several atomic layers, within which the elastic modulus varies continuously from the top layer to the inside region through a transition zone, ${ }^{24}$ while the continuum model assumes a zero-thickness surface, across which the modulus should experience a jump from the inside to the surface layer. ${ }^{9,35,43}$ Therefore, the different definitions of nanowire's surface in theoretical and numerical models should be responsible for the deviation of the results, especially when the characteristic length of nanomaterials or nanostructures is relatively small.

In addition, based on the surface elasticity theory, a FE model was proposed by Feng et al. ${ }^{31}$ to predict the fundamental resonant frequency of a silicon cantilever nanowire. Compared with Fig. 3, the FE result quantitatively agrees

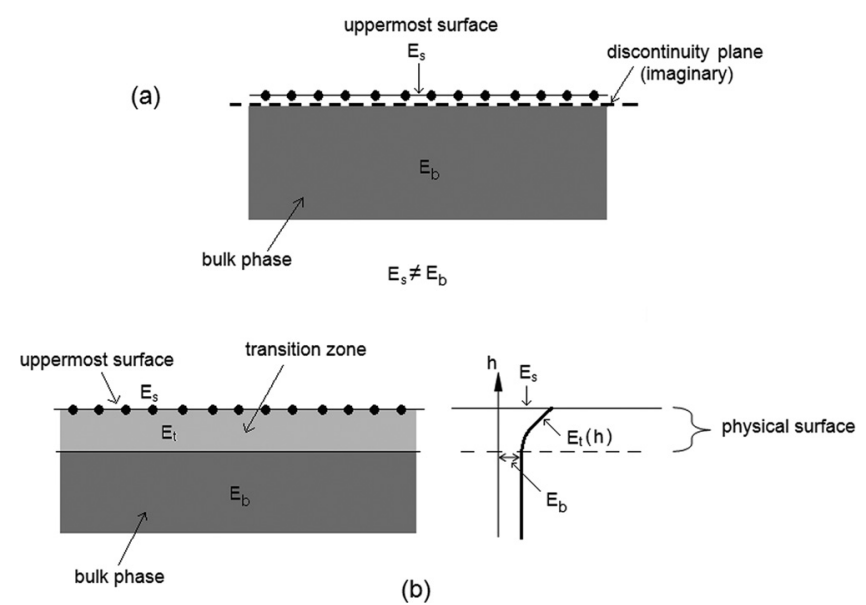

FIG. 4. Schematic of the definition of a nanowire's surface. (a) A zerothickness surface in theoretical models; (b) the surface layer connects continuously with the inside through a transition zone in the SCB model. ${ }^{24}$

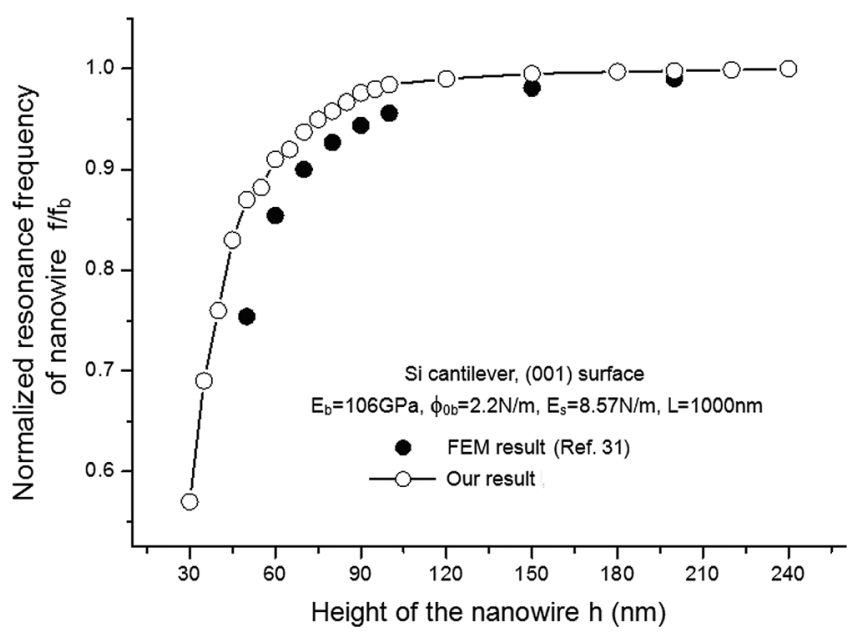

FIG. 5. Comparison of the resonant frequency between the theoretical prediction and the $\mathrm{FE}$ result ${ }^{31}$ for a silicon cantilever nanowire.

with our theoretical predictions as shown in Fig. 5, which demonstrates the fact that a zero-thickness surface assumption is adopted both in our theoretical model and in the FE one within the framework of the surface elasticity theory. ${ }^{31}$ When the height of nanowires is smaller than $100 \mathrm{~nm}$, deviation between the theoretical and numerical results is inevitable due to different considerations of the surface effect in nanomaterials. The surface elastic constants in the surface elasticity theory are independent of the sample size, while the surface relaxation parameter in the present model depends on it.

A recent numerical study based on density functional theory (DFT) simulations found that the elastic properties of metallic nanostructures exhibit not only the size dependent feature due to the surface effect but also a size-dependent oscillation arising from a quantum size effect (QSE). ${ }^{63}$ The quantum effect is a crucial attribute of a nanoscale system since the dangling bonds on the free surface of nanomaterials would induce obvious variations in atoms besides the bond length change ${ }^{52}$ However, such an effect at atomic scales is difficult to be characterized by continuum approaches and MD simulations. Further investigations on the mechanical behavior of nanomaterials need to consider quantum effects.

\section{CONCLUSIONS}

Based on the recently developed continuum theory for nanomaterials, ${ }^{43}$ the resonant vibration behavior of nanowires with different boundary conditions is investigated. The theoretical prediction of fundamental resonant frequency exhibits a consistent size-dependence with the existing numerical result, i.e., stiffening of a fixed-fixed nanowire while softening of a cantilever one for a decreasing characteristic length. Such a varying trend can be further enhanced by increasing the aspect ratio of nanowires. The result in the present paper should be helpful for the design of resonators and sensors with an aim of controllable mechanical performances.

\section{ACKNOWLEDGMENTS}

The work reported here was supported by NSFC through Grant Nos. 11372317 and 11125211, the Nano- 
project (2012CB937500), and the CAS/SAFEA International Partnership Program for Creative Research Teams.

${ }^{1}$ R. E. Miller and V. B. Shenoy, Nanotechnology 11, 139 (2000).

${ }^{2}$ H. Y. Liang and M. Upmanyu, Phys. Rev. B 71, 241403 (2005).

${ }^{3}$ G. Y. Jing, H. L. Duan, X. M. Sun, Z. S. Zhang, J. Xu, Y. D. Li, J. X. Wang, and D. P. Yu, Phys. Rev. B 73, 235409 (2006).

${ }^{4}$ S. Cuenot, C. Fretigny, S. D. Champagne, and B. Nysten, Phys. Rev. B 69, 165410 (2004).

${ }^{5}$ Y. X. Chen, B. L. Dorgan, D. N. Mcllroy, and D. E. Aston, J. Appl. Phys. 100, 104301 (2006)

${ }^{6}$ E. C. C. M. Silva, L. M. Tong, S. Yip, and K. J. van Vliet, Small 2, 239 (2006).

${ }^{7}$ H. Sadeghian, C. K. Yang, J. F. L. Goosen, A. Bossche, U. Staufer, P. J. French, and F. Van Keulen, J. Micromech. Microeng. 20, 64012 (2010).

${ }^{8}$ E. Celik, I. Guven, and E. Madenci, Nanotechnology 22, 155702 (2011).

${ }^{9}$ M. E. Gurtin and A. I. Murdoch, Arch. Ration. Mech. Anal. 57, 291 (1975).

${ }^{10}$ M. E. Gurtin and A. I. Murdoch, Int. J. Solids Struct. 14, 431 (1978).

${ }^{11}$ D. J. Steigmann and R. W. Ogden, Proc. R. Soc. London, Ser. A 453, 853 (1997).

${ }^{12}$ J. He and C. M. Lilley, Nano Lett. 8, 1798 (2008).

${ }^{13}$ P. Chhapadia, P. Mohammadi, and P. Sharma, J. Mech. Phys. Solids 59, 2103 (2011).

${ }^{14}$ M. S. Chiu and T. Y. Chen, Physica E 44, 714 (2011).

${ }^{15}$ X. F. Li, H. Zhang, and K. Y. Lee, Int. J. Mech. Sci. 81, 120 (2014).

${ }^{16}$ S. H. Park, J. S. Kim, J. H. Park, J. S. Lee, Y. K. Choi, and O. M. Kwon, Thin Solid Films 492, 285 (2005).

${ }^{17}$ W. K. Chan and T. Y. Zhang, J. Appl. Phys. 107, 023526 (2010).

${ }^{18}$ P. Mohammadi and P. Sharma, Appl. Phys. Lett. 100, 133110 (2012).

${ }^{19}$ D. Georgakaki, O. G. Ziogos, and H. M. Polatoglou, Phys. Status Solidi A 211, 267 (2014)

${ }^{20}$ H. G. Craighead, Science 290, 1532 (2000).

${ }^{21}$ H. L. Duan, Acta Mech. Solida. Sin. 23, 1 (2010).

${ }^{22}$ P. Xie, Q. H. Xiong, Y. Fang, Q. Qing, and C. M. Lieber, Nat. Nanotechnol. 7, 119 (2012)

${ }^{23}$ J. Llobet, M. Sansa, M. Gerboles, N. Mestres, J. Arbiol, X. Borrise, and F. Murano-Perez, Nanotechnology 25, 135302 (2014).

${ }^{24}$ H. S. Park and P. A. Klein, J. Mech. Phys. Solids 56, 3144 (2008).

${ }^{25}$ X. X. Li, T. Ono, Y. L. Wang, and M. Esashi, Appl. Phys. Lett. 83, 3081 (2003).

${ }^{26}$ C. Y. Nam, P. Jaroenapibal, D. Tham, D. E. Luzzi, S. Evoy, and J. E. Fischer, Nano Lett. 6, 153 (2006).

${ }^{27}$ K. B. Gavan, H. J. R. Westra, E. W. J. M. Van der drift, W. J. Venstra, and H. S. J. Van der zant, Appl. Phys. Lett. 94, 233108 (2009).

${ }^{28}$ C. Q. Chen, Y. Shi, Y. S. Zhang, J. Zhu, and Y. J. Yan, Phys. Rev. Lett. 96, 075505 (2006).
${ }^{29}$ Q. Q. Qin, F. Xu, Y. Q. Cao, P. I. Ro, and Y. Zhu, Small 8, 2571 (2012).

${ }^{30}$ H. S. Park, J. Appl. Phys. 103, 123504 (2008).

${ }^{31}$ Y. K. Feng, Y. L. Liu, and B. Wang, Acta Mech. 217, 149 (2011).

${ }^{32}$ P. A. T. Olsson and H. S. Park, J. Mech. Phys. Solids 60, 2064 (2012).

${ }^{33}$ P. Lu, H. P. Lee, C. Lu, and S. J. O. Shea, Phys. Rev. B 72, 085405 (2005).

${ }^{34}$ G. F. Wang and X. Q. Feng, Appl. Phys. Lett. 90, 231904 (2007).

${ }^{35}$ J. He and C. M. Lilley, Appl. Phys. Lett. 93, 263108 (2008).

${ }^{36}$ G. F. Wang and X. Q. Feng, J. Phys. D: Appl. Phys. 42, 155411 (2009).

${ }^{37}$ Q. L. He and C. M. Lilley, J. Appl. Phys. 112, 074322 (2012).

${ }^{38}$ M. S. Chiu and T. Y. Chen, Acta Mech. 223, 1473 (2012).

${ }^{39}$ Y. Zhang, L. J. Zhuo, and H. S. Zhao, Proc. R. Soc. A 469, 20130449 (2013).

${ }^{40}$ J. X. Wang, Z. P. Huang, H. L. Duan, S. W. Yu, X. Q. Feng, G. F. Wang, W. X. Zhang, and T. J. Wang, Acta Mech. Solida. Sin. 24, 52 (2011).

${ }^{41}$ V. B. Shenoy, Phys. Rev. B 71, 094104 (2005).

${ }^{42}$ C. W. Mi, S. Jun, D. A. Kouris, and S. Y. Kim, Phys. Rev. B 77, 075425 (2008).

${ }^{43}$ S. H. Chen and Y. Yao, ASME J. Appl. Mech. 81, 121002 (2014).

${ }^{44}$ Y. Yao, Y. C. Wei, and S. H. Chen, Surf. Sci. 636, 19 (2015).

${ }^{45}$ Y. Yao and S. H. Chen, "Surface effect in static bending nanowires predicted by an elastic theory for nanomaterials," Int. J. Solids Struct. (submitted).

${ }^{46}$ H. J. Wasserman and J. S. Vermaak, Surf. Sci. 22, 164 (1970).

${ }^{47}$ F. H. Streitz, R. C. Cammarata, and K. Sieradzki, Phys. Rev. B 49, 10699 (1994).

${ }^{48}$ R. Lamber, S. Wetjen, and N. I. Jaeger, Phys. Rev. B 51, 10968 (1995).

${ }^{49}$ B. Medasani, Y. H. Park, and I. Vasiliev, Phys. Rev. B 75, 235436 (2007).

${ }^{50}$ W. D. Nix and H. Gao, Scr. Mater. 39, 1653 (1998).

${ }^{51}$ Z. P. Huang and J. Wang, Acta Mech. 182, 195 (2006).

${ }^{52}$ C. Q. Sun, Prog. Mater. Sci. 48, 521 (2003).

${ }^{53}$ G. Ouyang, X. Tan, and G. W. Yang, Phys. Rev. B 74, 195408 (2006).

${ }^{54}$ S. P. Timoshenko and J. M. Gere, Mechanics of Materials (Van Nostrand Reinhold Co., New York, 1972).

${ }^{55}$ L. G. Zhou and H. C. Huang, Appl. Phys. Lett. 84, 1940 (2004).

${ }^{56}$ W. X. Zhang, T. J. Wang, and X. Chen, Int. J. Plast. 26, 957 (2010).

${ }^{57}$ T. Y. Chen and M. S. Chiu, Mech. Mater. 43, 212 (2011).

${ }^{58}$ F. Song, G. L. Huang, H. S. Park, and X. N. Liu, Int. J. Solids Struct. 48, 2154 (2011).

${ }^{59}$ D. H. Hodges and G. A. Pierce, Introduction to Structural Dynamics and Aeroelasticity, 2nd ed. (Cambridge University Press, New York, 2011).

${ }^{60}$ H. W. Sheng, M. J. Kramer, A. Cadien, T. Fujita, and M. W. Chen, Phys. Rev. B 83, 134118 (2011).

${ }^{61}$ L. Vitos, A. V. Ruban, H. L. Skriver, and J. Kollar, Surf. Sci. 411, 186 (1998).

${ }^{62}$ J. K. Diao, K. Gall, and M. L. Dunn, J. Mech. Phys. Solids 52, 1935 (2004).

${ }^{63}$ M. Liu and F. Liu, Nanotechnology 25, 135706 (2014). 